\title{
Introduction to the supratentorial cerebral arteriovenous malformation video supplement
}

\author{
Robert M. Starke, MD, MSc, ${ }^{1}$ Felipe C. Albuquerque, MD, ${ }^{2}$ and Michael T. Lawton, MD $^{3}$ \\ ${ }^{1}$ Neurosurgery and Radiology, University of Miami, Florida; ${ }^{2}$ Department of Neurological Surgery, Barrow Neurological Institute, \\ Phoenix, Arizona; and ${ }^{3}$ Department of Neurological Surgery, University of California, San Francisco, California \\ It is with great pleasure that we present this Neurosurgical Focus video supplement on supratentorial cerebral arterio- \\ venous malformations (AVMs). We were privileged to view a remarkable number of outstanding videos demonstrating \\ current state-of-the-art management of brain AVMs using endovascular and microsurgical modalities. Careful and critical \\ review was required to narrow down the submitted videos to a workable volume for this supplement, which reflects the \\ excellent work being done at multiple centers with these lesions. \\ This issue consists of videos that represent modern microsurgical and neuroendovascular techniques for the treatment \\ of supratentorial cerebral AVMs. The videos demonstrate cutting-edge therapies as well as standard ones, which will be \\ valuable to both novice and expert neurointerventionists and neurosurgeons. We are honored to be involved with this \\ project and proud of its content and expert authors. We believe you will enjoy the video content of this supplement and \\ hope that it will raise the collective expertise of our community of AVM surgeons.
}

KEYWORDS AVM; cerebral arteriovenous malformation; supratentorial; video 